\title{
Chromatic Line Confocal Sensor Technology in High-Speed 3D and Deep Depth of Focus 2D Imaging Applications
}

Juha Saily ${ }^{1}$

${ }^{1 .}$ FocalSpec, Inc., Cumming, GA, USA.

In the late 2000's researchers at Technical Research Center of Finland (VTT) invented a new method for 3D surface imaging at sub-micron resolution [1]. This method, Line Confocal Imaging (LCI), is based on freezing a continuous line of wavelength reflections in visible spectrum from more than 2000 lateral points at the same time. In 2009, FocalSpec Ltd. was founded as a spin-off from VTT to further develop and commercialize the LCI technology.

LCI can be used in 3D imaging of surfaces, transparent materials and multi-layered structures in various analytical, metrology and inspection applications. Imaging results from LCI systems can be used in determining a sample's 3D form and dimensions; surface topography, roughness and texture; thickness, flatness, volume, etc. An additional feature of this technology is its capability to simultaneously capture gray-scale 2D images with large depth of focus that covers the sensor's entire $\mathrm{z}$ range.

Figure 1 shows the LCI sensor models that are currently in production. Each sensor has two front lenses, one for its transmitter and another for the receiver. Figure 2 depicts the sensor's operating principle. The transmitter has a light source that emits white light containing all visible wavelengths. An optical assembly separates the light into wavelengths and focuses a horizontal line of each color at a different distance from the sensor, forming a focal plane (Figure 3). Depending on the vertical position of the imaged surface within the plane, corresponding wavelengths from 2048 lateral points are reflected back to the sensor's receiver. The receiver's spectral camera captures wavelength and intensity information from each point to form related height profile and gray-scale lines. When the surface is moved in front of the sensor (Figure 4), a 3D point cloud and 2D gray-scale image are generated from the scanned area line by line. The resulting data can be processed, analyzed and reported with various 3D surface analysis and image processing software packages.

Depending on the imaged material, surface type and resolution requirements, three sensor models have been made available. The sensor with highest resolution offers vertical $(\mathrm{z})$ resolution of $100 \mathrm{~nm}$ and $\mathrm{z}$ range of $1.00 \mathrm{~mm}$. The next model up in size has $\mathrm{z}$ range of $2.80 \mathrm{~mm}$ and $\mathrm{z}$ resolution of $0.55 \mu \mathrm{m}$. The largest model has $\mathrm{z}$ range of $5.50 \mathrm{~mm}$ and $\mathrm{z}$ resolution of $0.98 \mu \mathrm{m}$. Lateral resolution in measurement line direction (x) varies from 2.20 to $8.00 \mu \mathrm{m}$, depending on the sensor model. Y resolution depends on the line spacing which also affects the surface motion speed in the application. The length of the sensors' measurement line is from 4.50 to $16.40 \mathrm{~mm}$ [2]. The sensors can be integrated in both laboratory and realtime in-production systems as they can operate at the speed of up to 4000 lines per second [3]. This results in data acquisition rate of over 8 million $3 \mathrm{D}$ surface points per second.

LCI works well in applications that require high-speed imaging of challenging materials at sub-micron resolution. Materials with highly reflective, mirror-like, high-contrast or transparent surfaces are ideal for this method. Surface colour does not affect the imaging results. Large numerical aperture and tolerance for surface angle variation allow for imaging of slopes with fully specular surfaces up to \pm 20 degrees from normal and almost \pm 90 degrees on diffuse surfaces. 
LCI can be used to image various materials such as plastics, metals, glass, ceramics, composites and paper. Etched, embossed, molded, extruded, micro-machined and stamped products are all suitable for the method. New promising applications include medical devices, printed electronics, microfluidic devices and dispensed fluids as they often have specular or transparent surfaces. One of the first applications using deep depth of focus 2D LCI imaging is the inspection of heat seal integrity in sterile medical packages.

Even though most LCI sensors and systems are currently being used in research and development, quality control and failure analysis of manufactured parts, assemblies and continuous products, the technology also has a wide range of potential uses in materials science and biomedical applications.

Figure 5 shows a 3D LCI scan of a water drop (height approx. $350 \mu \mathrm{m}$ ) on black anodized aluminum surface. Figure 6 depicts a detail of a 2D scan from $1.5 \mathrm{~mm}$ thick transparent plastic sheet with two horizontal scratches on the surface facing the sensor and a short vertical scratch on the other side.

[1] Antti Kemppainen et al, Research and development activities in printed intelligence (2009) pp. 74-76. [2] Juha Saily et al, Flex conference proceedings (2017) p. 15-4.

[3] Juuso Hautala, Use of optical measurement unit in development of extrusion process (2016) pp. 1-70.

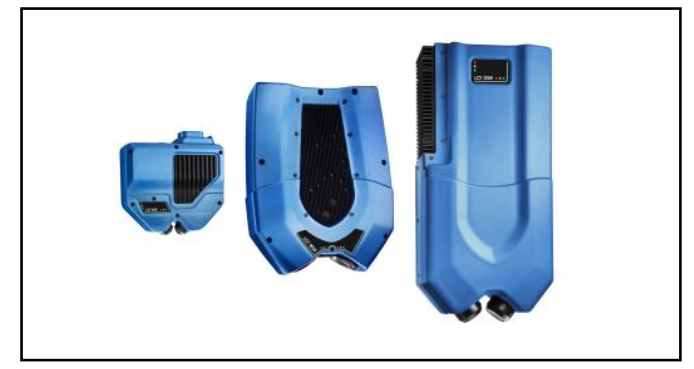

Figure 1. Line confocal imaging sensors

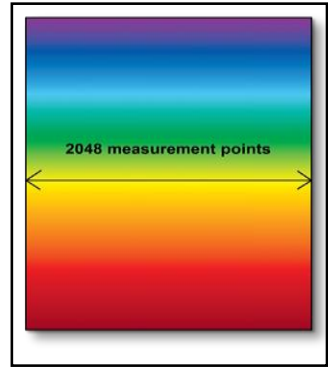

Figure 3. Focal plane

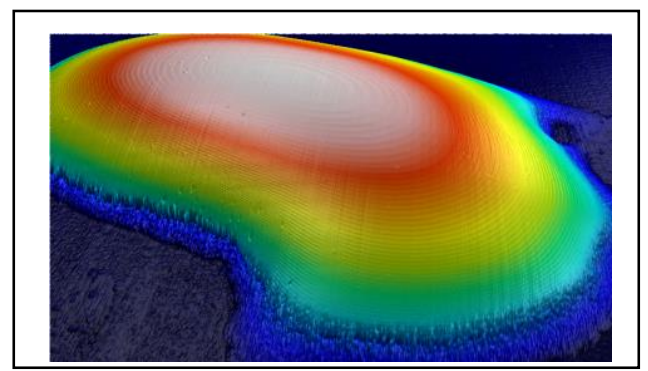

Figure 5. Water drop on aluminum surface

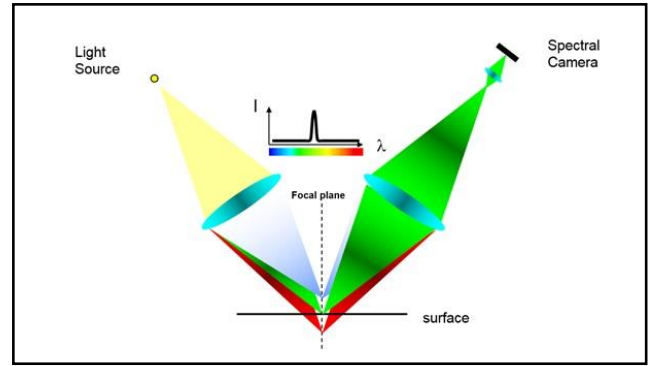

Figure 2. Line confocal imaging principle

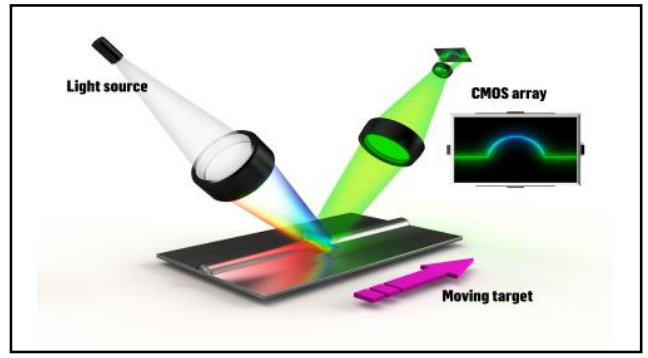

Figure 4. 3D imaging of an area

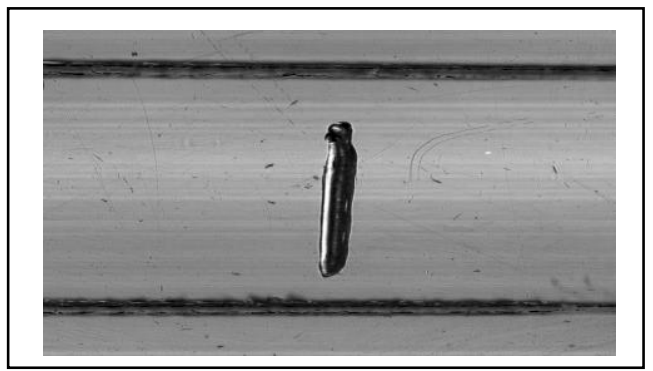

Figure 6. Deep depth of focus 2D scan 